\title{
SILVER NANOPARTICLES AND COCONUT OIL INCORPORATED BIOPOLYMER BASED ELECTROSPUN NANOFIBERS FOR WOUND DRESSING
}

\author{
SUBHASHREE SAHOO ${ }^{1}$, JOGABRATA TRIPATHY ${ }^{1}$, AFRASIM MOIN ${ }^{2}$, MEENAKSHI S. ${ }^{3}$, SIDDARAMAIAH ${ }^{4}$, D. V. \\ GOWDA $^{1^{*}}$
}

1,*Department of Pharmaceutics, JSS College of Pharmacy, JSS Academy of Higher Education and Research, S. S. Nagara, Mysuru 570015, Karnataka, India, ${ }^{2}$ Department of Pharmaceutics, College of Pharmacy, University of Hail, PO Box 2440, Hail, Saudi Arabia, ${ }^{3}$ Department of Prosthodontics, JSS Dental College and Hospital, JSS Academy of Higher Education and Research, S. S. Nagara, Mysuru 570015, Karnataka, India, ${ }^{4}$ Department of Polymer Science and Technology, S. J. College of Engineering, JSS Science and Technology University, Mysuru 570006, Karnataka, India

*Email: dvgowda@jssuni.edu.in

Received: 18 Nov 2020, Revised and Accepted: 09 Jan 2021

\section{ABSTRACT}

Objective: The main aim of this study was to develop and evaluate the nanofiber loaded with coconut oil and silver nanoparticles (Ag NPs) for the treatment of wound healing by the electrospun method.

Methods: The nanofibers have been created using the reduced form of silver nanoparticles and coconut oil along with Eudragit L-100 by the electrospun method. The presence of coconut oil and chemical interaction was determined by the FTIR method. XRD was made to evaluate the crystalline nature of AgNPs and Eudragit L-100. TEM was carried out to show the presence of AgNPs on the surface of nanofibers and SEM represents the diameter of the fiber. The antibacterial activity of nanofibers was carried out using a disk diffusion assay.

Results: The diameter of the fibers was diminished by the excess of AgNPs in the fibers, while it increases by the coconut oil concentration, enhancing the nanofiber's hydrophilicity. FTIR spectroscopy was found in the range of coconut oil at $3553 \mathrm{~cm}^{-1}$ for 0 - $\mathrm{H}$ stretch, $1365 \mathrm{~cm}^{-1}$, and 1240 $\mathrm{cm}^{-1}$ for the C-O stretch of ester groups. The diffraction peaks at $2 \theta$ of $38.5^{\circ}, 44.6^{\circ}$, and $64.7^{\circ}$, in the XRD spectra of nanofiber, changed with silver NP affirming the total decrease of Ag salt. The bactericidal activity has been carried out between Escherichia coli and Staphylococcus aureus showing zones of inhibition of $20.0 \pm 0.2 \mathrm{~mm}$ and $14.8 \pm 0.4 \mathrm{~mm}$, exhibiting excellent bactericidal characteristics for wound healing.

Conclusion: The formulated nanofibers were obtained to offer protection against external agents and help in the regeneration of new tissue.

Keywords: Polymeric nanofiber, Wound healing, Electrospun method, Escherichia coli, Staphylococcus aureus

(C) 2021 The Authors. Published by Innovare Academic Sciences Pvt Ltd. This is an open access article under the CC BY license (https://creativecommons.org/licenses/by/4.0/) DOI: https://dx.doi.org/10.22159/ijap.2021v13i2.40291. Journal homepage: https://innovareacademics.in/journals/index.php/ijap

\section{INTRODUCTION}

Wounds are brought about by interruption of typical tissue-lined anatomical epithelial obstructions. Wound healing is a perplexing procedure, including an assortment of occasions in various tissues and cell lines [1]. Due to the high surface region to-volume proportion and explicit compound and physical properties, silver nanoparticles have risen as novel antimicrobial operators. Silver nanoparticles can be consolidated into biomaterials and platforms to make polymeric nanofibers, which can help twisted recuperating through their antimicrobial and antibacterial properties [2, 3].

Electrospinning nanofibers are light-weight, little distance across filaments going from a couple of lengths to more than $100 \mathrm{~nm}$. Nanofibers have incredible potential for an assortment of utilizations, for example, composites, filtration, biomaterials, and so forth. These fibers are regularly utilized as it is being able to expand water dissolvability, biorecognition, and biocompatibility. Main strategies used to deliver nanofibers, for example, electrospinning, centrifugal spinning, pressurized gyration, model synthesis phase separation, phase assembly [4]. In any case, electrospinning is viewed as the easiest and best methodology for nano-to microscale strands creation [5].

In this assessment, we exhibited the dressings included with electrospun nanofibers containing coconut oil and silver particle of nano-size have feasible antiseptic characteristics because of their symbiotic development [6]. In this appraisal, it showed the dressings included with electrospun nanofibers containing coconut oil and silver nanoparticle have achievable bactericidal properties due to their symbiotic improvement. Biopolymer-based nanofibers got together with coconut oil and silver nanoparticles to avoid issues such as low adaptability of the dressing, wound area significance, and low-run bactericidal action. The resulted nanofiber demonstrated the closeness of both coconut oil and silver nanoparticles in the production of the nanofiber. Scanning electron microscopy (SEM) studies showed that the coconut oil and silver nanoparticless produce unfavorable activity in controlling the width of the fiber. Even the proximity of coconut oil may improve the rigid idea of the strands through basic dispersal and immersion into the thermal softening plastic particles related polymers. Cytoconformity resulted in improving the advancement and increase of fibroblasts of the fiber in a segment subordinate way, with a limited amount of coconut oil with silver nanoparticles $[7,8]$.

\section{MATERIALS AND METHODS}

\section{Materials}

Silver nitrate $\left(\mathrm{AgNO}_{3}\right)$ was obtained from SRL Lab Pvt. Ltd. Mumbai, India, and coconut oil Cocos nucifera (CO) were procured from Ambal Import and Export, Tamil Nadu, India. $\mathrm{N}, \mathrm{N}-$ dimethylformamide (DMF) were obtained from ACE Rasayan, India, and tetrahydrofuran (THF) was purchased from TCI Chemicals, India. The agar broth media were obtained from Ranbaxy Lab. Pvt. Ltd., Bangalore, India, and Eudragit L-100 were purchased from Quest Int. Bogles. Gram-ve E. coli and Gram+ve S. aureus were obtained from the Faculty of Lifescience, JSS Academy of Higher Education, and Research. All chemicals used in this study were experimentally qualified and used without further purgation.

\section{Methods}

\section{Preparation of electrospun nanofiber dressings}

Nanofiber was created using coconut oil and silver nanoparticles with the help of an electrospinning framework. To begin with, the Ag NPs were readied utilizing the decrease of silver nitrate salt by maturing it in $\mathrm{N}, \mathrm{N}$-dimethylformamide $(30$ sections as needed for 
electrospinning) for $72 \mathrm{~h}$ at $20-30{ }^{\circ} \mathrm{C}$ and observing the shading difference of the silver scattering [9]. After $72 \mathrm{~h}$, silver salt was completely deduced into silver metal, showed a major modification in color dispersion from slight golden to dark black. The nanofiber was created using the decreased (reduction process) type of silver nanoparticles and coconut oil along with Eudragit L-100 in electrospinning. It was then dissipated in THF (70 sections) by steady mixing using a magnetic stirrer (Whirlmatic Spectra Lab., Mumbai, India) at $400 \mathrm{rpm}$ [10]. At first, various convergences of $\mathrm{Ag}$ NPs $(0,2,4,6$, and 8\%) (DMF) and the sample in THF were combined in the proportion of 70:30 alongside various formulations of coconut oil $(0,4,8,12$, and $16 \%)$. Each of the resultant scatterings was then stacked into three dispensable syringes with an ostensible inward needle measurement of $0.82 \mathrm{~mm}$. Subsequently, $15 \mathrm{ml}$ of the readied arrangement, the syringes were put in the stands in the electrospinning gathering. The strands were gathered at a point of $115^{\circ} \mathrm{C}$ comparative in the flat plane, confronting the keeping anode. The inner syringe needle was related to a positive power supply. The needle tip points were relatively joined together with the help of an extra copper loop that provided sufficient current to all syringes. It was carried out at a voltage of $20 \mathrm{kV}$, point tip-to-gatherer of $20 \mathrm{~cm}$, a stream pace of $0.8 \mathrm{ml} / \mathrm{h}$, a temperature of $27{ }^{\circ} \mathrm{C}$, appropriate dampness of $50 \%$, and a drum collector speed of $120 \mathrm{rpm}$, which were kept up during electron spin. After that, the nanofiber was accumulated on the Al sheet collapsed around the metal drum gatherer, having outside separation crosswise over $5 \mathrm{~cm}$. In general, $35 \mathrm{ml}$ of the polymer arrangement was collected on the Al sheet, preserved in airtight containers, and stored for future use [11].

Table 1: Formulations with different concentration of AgNPs and coconut oil

\begin{tabular}{llll}
\hline Formulations & AgNPs & CO & Fiber formation \\
\hline F1 & 0 & 0 & B \\
F2 & 2 & 4 & B \\
F3 & 4 & 8 & B \\
F4 & 6 & 12 & B \\
F5 & 8 & 16 & A \\
\hline
\end{tabular}

${ }^{*}$ A-Good, B-Poor; AgNPs= Silver Nanoparticles; CO= Coconut oil

\section{Morphological analysis}

Nanofibers are dismembered with the help of Scanning Electron Microscopy (SEM) (Zeiss EVO LS 15, Smart SEM 5.05, and Germany). The models were vacuum-dried for 25 mins to get free from moistness. The photos of the nanofiber were collected using an animating voltage of $5 \mathrm{kV}$, at $1000 \mathrm{x}$, and 5000x [10].

To detect the location of raw AgNPs on the surface of the membrane, transmission electron microscopy (TEM) was performed. During the combustion method, the samples for the TEM analysis were precisely packed. In short, the fibers extracted from the needle were accurately stored on $3 \mathrm{~mm}$ copper grids by keeping the TEM grids between the needle and the collector for $30 \mathrm{~s}$ with point forceps. At $10,000 \times$ and $20,000 \times$ magnifications and an operating voltage of 200 $\mathrm{kV}$, the TEM micrographs were captured. Both product was dried at $37^{\circ} \mathrm{C}$ for $2 \mathrm{~h}$ prior to the TEM evaluation.

\section{Chemical investigations}

FTIR is utilized to decide the nearness of coconut oil or compound association which happened in the course of nanofiber manufacturing by electrospinning procedure. The range is documented somewhere in between 650 and $4000 \mathrm{~cm}^{-1}$ utilizing the FTIR-8400S, Shimadzu, Kyoto, Japan FTIR spectrometer at a goal of $8 \mathrm{~cm}^{-1}$ and a filtering pace of 32 [12]. Using an X-ray diffractometer with $\mathrm{Cu} \mathrm{K} \alpha$ radiation and a Bragg angle ranging from $5^{\circ}$ to $80^{\circ}$, and the phase, purity and crystallinity of the Ag NPs and polyurethane is calculated [13, 14].

\section{In vitro cytotoxicity tests}

Cytotoxicity of fiber wearing with changing centralizations of coconut oil and silver nanoparticles was surveyed using MTT examination on
Chicken embroy fibroblasts (CEFs). The test depends upon the decline 3-(4,5-dimethyl thiazol-2-yl)-2,5-diphenyltetrazolium bromide to purple form using precious stones with mitochondrial oxides available for alive cell. Before carrying out the analysis of CEFs was detached from a 10-day-old chicken early life form after removing from eggs and removing made farthest focuses. Undeveloped organisms cleaved with careful edge and a while later washed by $1.2 \mathrm{ml}$ hot trypsin trailed forbringing forth at $36.5{ }^{\circ} \mathrm{C}$. As such, they ought to be subtracted by the model absorbance $[15,16]$.

\section{Cell auxiliary study of nanofibers scaffold}

Connection with CEF of tangles as wound-dressing material is concentrated with research capacity of fiber mats that help fibroblast connection, development, relocation, with expansion since fibroblasts are principle cells to interact with Extra Cellular Matrix (ECM) (platform) at the time of recovery. To some things up, $100 \mu \mathrm{l}$ of Dulbecco's Modified Eagle's Medium (DMEM) consisting CEFs are refined over 9-mm-measurement round about mats of electrospun nanofibers that were in 95-well plates for $7 \mathrm{~d}$ [16].

\section{Determination of antibacterial activity}

Disinfectantaction of electrospun nanofibers of Eudragit L-100/silver $\mathrm{NPs}$ /coconut oil is performed for Gram-ve E. coli and Gram+ve S. aureus with plate dissemination test [17]. Disinfectant action of nanofiber mats consists of various centralizations of coconut oil and silver nanoparticles are contrasted with the electrospun nanofiber tangles for estimating zones of restraint. To begin with, the nanofiber mats are stripped off from aluminum foil. All analyses are performed thrice and outcomes are documented as mean [18].

\section{RESULTS AND DISCUSSION}

\section{Fibers morphology and diameter distribution}

Eudragit L-100/silver NPs/coconut oil nanofibers are evaluated to develop as viable disinfectant injury dressing materials. For deciding the correct convergence of Eudragit L-100 needed to shape free strands, a few preliminary trials were rehashed while changing various frameworks (e. g., voltage, stream amount, needle to gather separation mass of polymer and centralizations for silver nitrate with coconut oil) [19]. At the time of screening tests, strands of assorted morphologies having uncommon examples of combination, consisting of mutilated, the two morphologies, are watched. Ideal situations that brought about dot free filaments were chosen: Eudragit L-100 grouping of $3 \mathrm{gm}$ and dissolvable proportion of 90:10 (THF: DMF). Chosen group of Silver Nanoparticles, framed by lessening silver nitrate in co-solvent, needed for electrospinning found to be in the scope of $1-5 \%$. Previously the value of the silver particles framed from silver salt are considered to diminish the consistency of Eudragit fixations. Correspondingly, the centralization of coconut oil chose for the composite fiber was in the scope of $5-15 \%$ as for the Eudragit L100 focus. Accordingly, the measure of coconut was constrained to $15 \%$ as for the polymer utilized for electrospinning the arrangements. The SEM pictures of relict's examples are without globule with thick nearness of filaments with controlled porosity prevailed $[9,10]$.

\section{Chemical characterizations}

The FTIR studies have been studied on biopolymer fiber to probe the interactions between the components of the nanofiber. FTIR spectra are displayed in fig. 4 and the existence of coconut oil in the biopolymer fiber that has been affirmed by FTIR spectroscopy was found in the range of coconut oil at $3553 \mathrm{~cm}^{-1}$ for $\mathrm{O}-\mathrm{H}$ stretch. The strong band was found at $1740 \mathrm{~cm}^{-1}$ symbolizing the presence of the carbonyl group. The nearness of the ester segments is additionally affirmed by the group at 1365 and $1240 \mathrm{~cm}^{-1}$ which reflects the C-O stretch of ester groups. In the range of Eudragit L-100 nanofiber, the trademark minor wide top at $3467 \mathrm{~cm}^{-1}$ [19]. These outcomes assert the nearness of coconut oil or its segments in electrospun filaments.

The nearness of silver NPs in nanofiber was supported by X-ray diffraction studies. The diffraction peaks at $2 \theta$ of $38.5^{\circ}, 44.6^{\circ}$, and $64.7^{\circ}$, which compare to the silver, are available in the XRD spectra of nanofiber changed with silver NP. These results affirmed the total decrease of Ag salt by DMF to silver NP and the joining of silver NP into the fiber engineering $[20,21]$. 


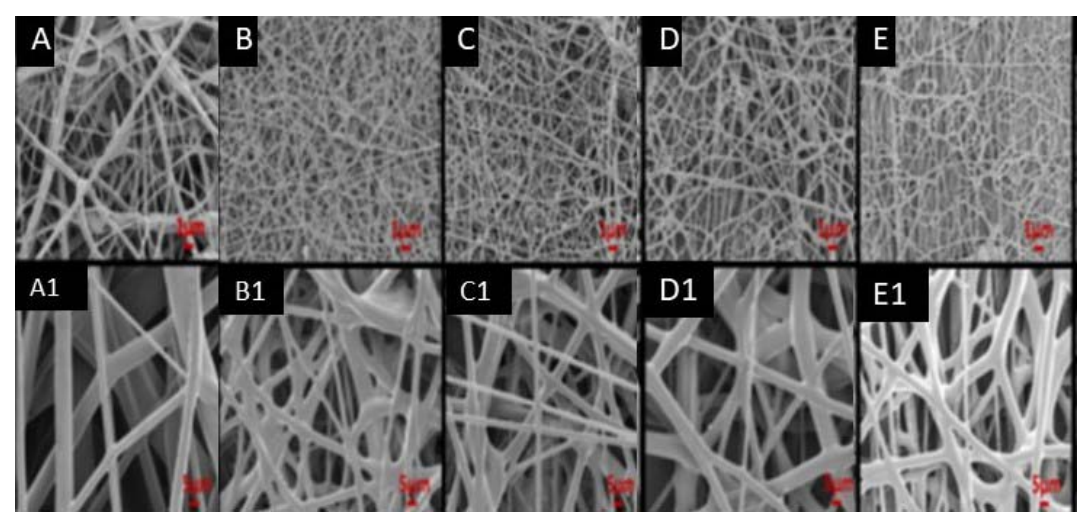

Fig. 1: SEM photomicrographs of Eudragit L-100/silver NPs/coconut oil fibers showing the morphologies of (A) Eudragit L-100, (B) EU+4\% CO/2\% AgNPs, (C) EU+8 \% CO/4\% Ag NPs, (D) EU+12\% CO/6\% Ag NPs, and (E) EU+16\% CO/8\%Ag NPs taken at 1000x magnification. (A1)-(E1) SEM photomicrographs taken at 5000x, magnification. ${ }^{* A g N P s}=$ Silver Nanoparticles; $\mathrm{CO}=\mathrm{Coconut}$ oil; EU= Eudragit L-100

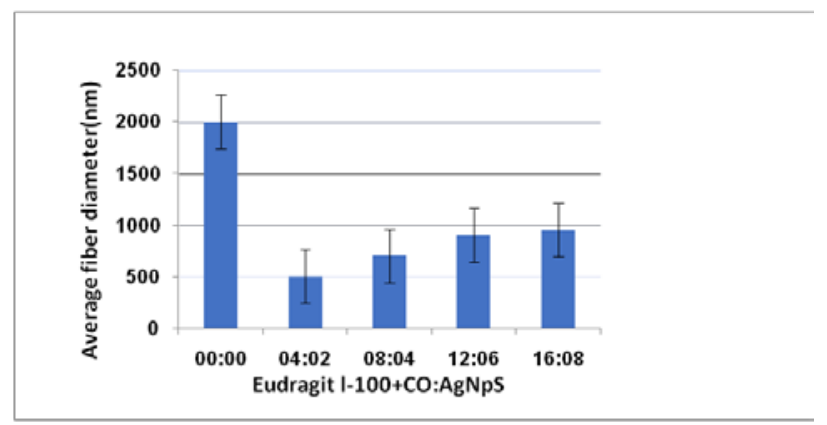

Fig. 2: Average diameter of Eudragit L-100 fiber with coconut oil and AgNPs contents showing a rise in fiber diameter with increasing concentration of Ag NPs in the electrospun nanofibers. *AgNPs= Silver Nanoparticles; CO= Coconut oil, $\operatorname{mean} \pm \mathrm{SD}(\mathrm{n}=3)$

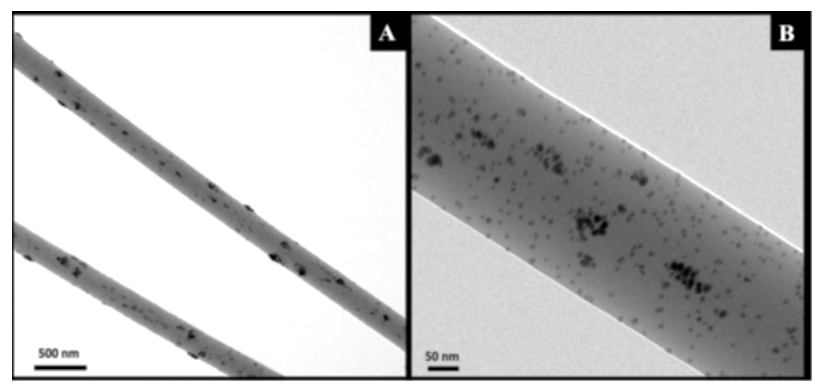

Fig. 3: TEM photomicrographs of Eudragit L-100/CO/AgNPs composite nanofibers

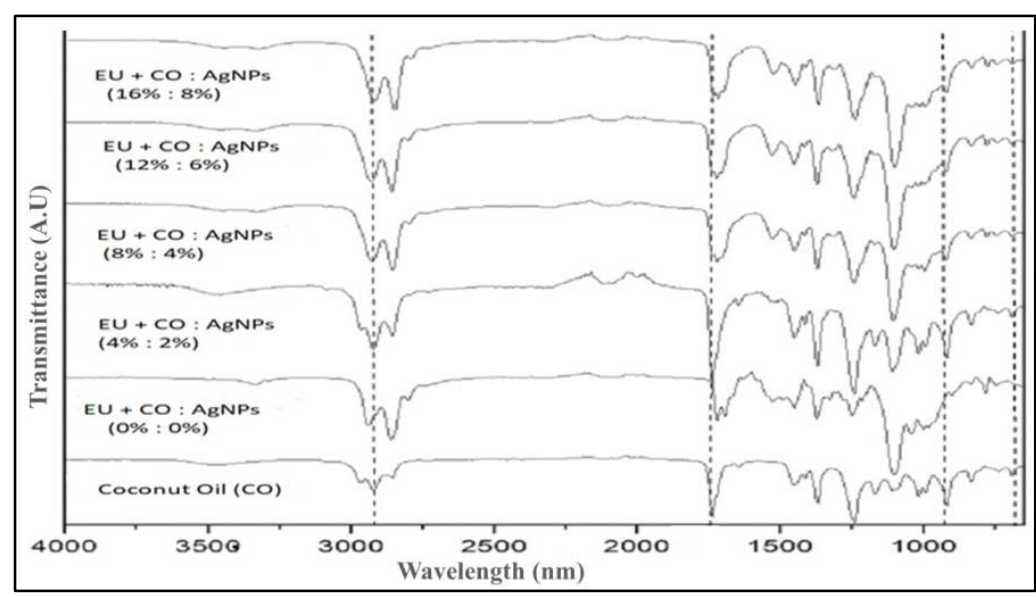

Fig. 4: FTIR spectra of electrospun fibers of Eudragit L-100/CO/AgNPs composite nanofibers with different amounts of CO and AgNPs content 


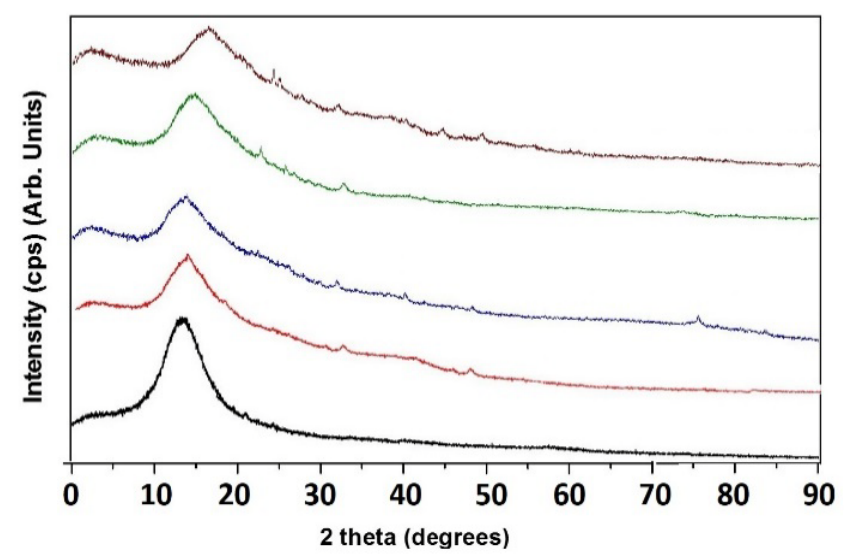

Fig. 5: XRD profiles of electrospun fibers of Eudragit L-100 containing different amounts of $\mathrm{CO}$ and AgNPs

\section{Hydrophilicity test}

The water holding capacity of nanofiber mats is evaluated by estimating surface point since water holding capacity assumes a significant job with biocompatibility, particularly at a time when nanofibers are utilized for in vivo uses. Since the filaments were proposed for the application of wound dressings, they ought to be in a specific level of water holding capacity for sticking with the surface of the wound. The contact edge tumbled from $72 \pm 3^{\circ}$ with unblemished fibers mat for $36 \pm 2.8^{\circ}$ for Eudragit L-100 consisting most elevated grouping of coconut oil and silver nanoparticles. Expanding centralization for coconut oil and silver nanoparticles diminish contact angle and consequently builds the water holding capacity [22].

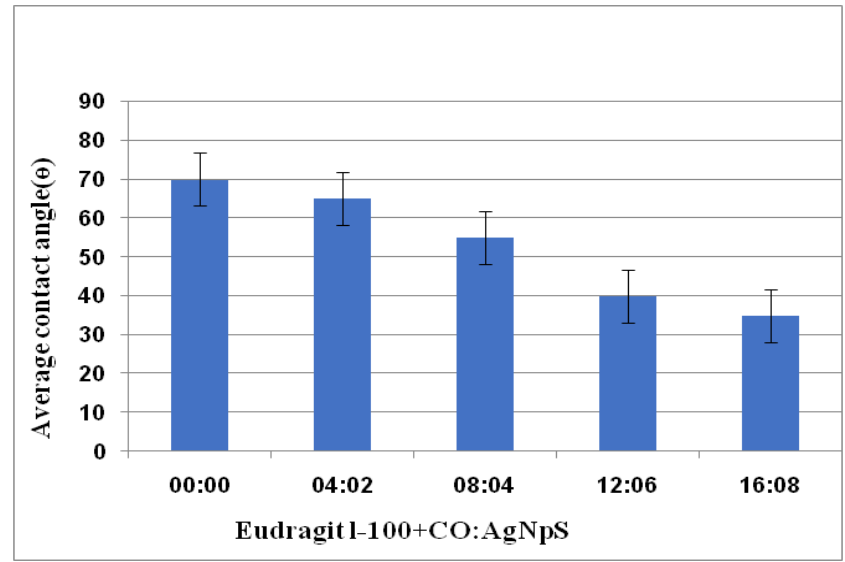

Fig. 6: Average contact angle of electrospun nanofiber of Eudragit L-100containing CO and AgNPs indicating an increase in the hydrophilicity of the mats along with rising in concentrations of CO and Ag NPs, *AgNPs= Silver Nanoparticles; $C O=C o c o n u t ~ o i l, ~ m e a n \pm S D ~(n=3)$

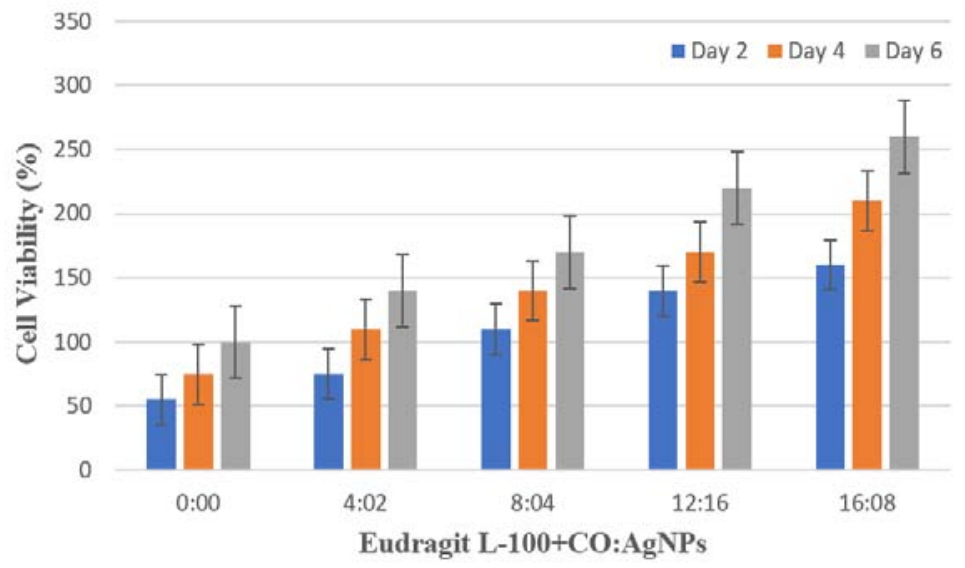

Fig. 7: Cell viability of Eudragit L-100 nanofiber with different amounts of CO and AgNPs. With the increased concentration of Ag NPs and CO, up to a minimal concentration of $16 \% \mathrm{CO} / 8 \% \mathrm{Ag}$ NPs, cell viability has been greatly improved, $*$ AgNPs $=$ Silver Nanoparticles; $\mathrm{CO}=$ Coconut oil, mean \pm SD $(n=3)$ 


\section{Biocompatibility of complex}

Biocompatibility with electrospun mat and CEF were assessed utilizing the MTT test. Since fibroblasts are significant cells that interact with harmed tissue, it is adverse, analyzing the practicality of the cells on the electrospun fiber. It shows the practicality of CEFs after refined within the sight of nanofiber tests for two, four, and sixday. Transformation for absorbance to the level of cell development relict's quantity of brooding time expanded, cell expansion expanded. Cell suitability essentially expanded as cells were refined in complex, recommending the coconut oil and silver nanoparticles improved cell multiplication. Looking at its feasibility of fibroblasts on electrospun nanofiber comprising of coconut oil and silver nanoparticles in strands upgraded practicality of fibroblasts up to the convergences of $16 \%$ coconut oil and $8 \%$ silver nanoparticles. Comparable outcomes were watched for 5 and $7 \mathrm{~d}$ of hatching, recommending the expansion for groupings of coconut oil and silver nanoparticles past $16 \%$ [23]

\section{Cellular attachment}

Cell connection activity was done to exhibit the impact of fiber morphology with the nearness of coconut oil and silver nanoparticles in connection, development, and multiplication of CEFs. This considered the cells are attached to electrospun nanofibers hatching in 6 d. The hydrophobicity of EudragitL-100 causes high pressure and subsequently lose surface among cells and the framework material. Therefore, the focused-on cells expect round morphological behavior, what's more, can't spread like fibroblasts nor experience ordinary development and expansion given the misfortune of contact with neighboring cells. Expanding the grouping of the coconut oil and silver nanoparticles, which alters the water holding capacity of nanofibers enables cells to reach the surface and proliferate through ECM. Not many fibroblasts were found in SEM micrography of an electrospun complex consisting of $16 \%$ coconut oil and $8 \%$ silver nanoparticles [16].

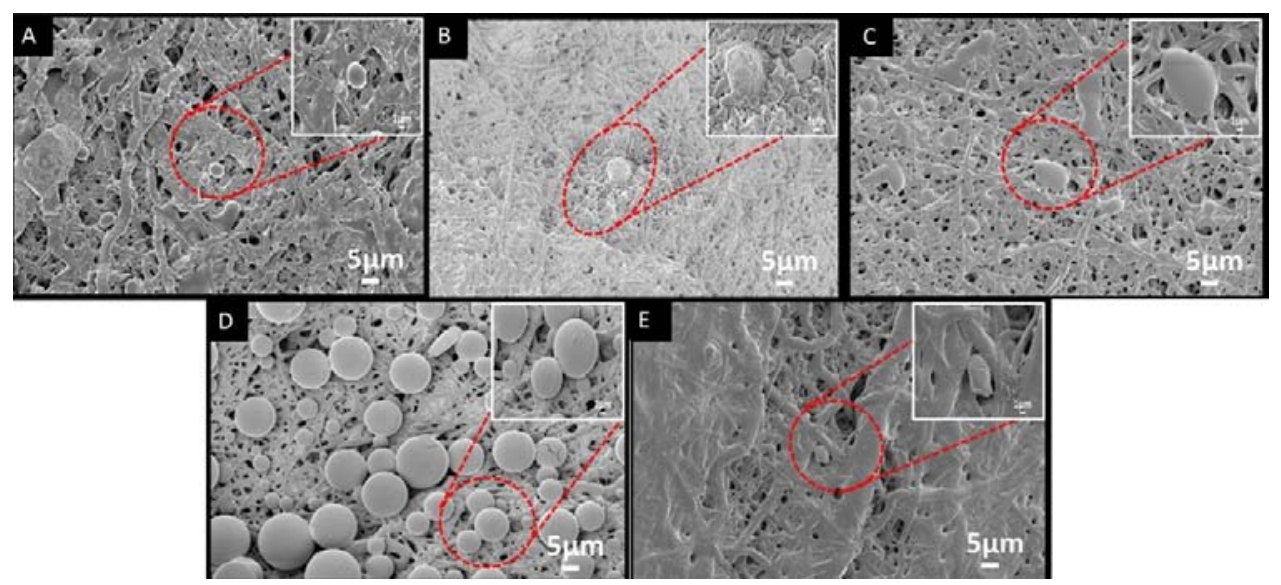

Fig. 8: SEM micrographs of fixed CEFs cultured on nanofibers with different amounts of AgNPs and CO. (A)Eudragit L-100, (B) EU+4\% CO/2\% Ag NPs, (C) EU+8\% CO/4\% Ag NPs, (D) EU+12\% CO/6\% Ag NPs, and (E) EU+16\% CO/8\% Ag NPs taken at 1000X magnification. Images showing cell morphology at $5000 \mathrm{X}$ magnification

\section{Bactericidal activity}

Disinfectant action of electrospun nanofiber mats and complex are exhibited between E. coli and S. aureus utilizing disk diffusion test; outcomes are exhibited, that relicts' immaculate Eudragit L-100 mats didn't hinder the development of strain. Conversely, the mats consisting of silver nanoparticles with coconut oil were successful to smother microbial development. Generally, the silver nanoparticles/coconut oil- consisting mats are progressively powerful against $E$. coli than between S. aureus showing bigger ZOIs [24]. Likewise, expanding convergences of coconut oil and silver nanoparticles brought about bigger ZOIs for the two strains because of the synergistic antibacterial movement of two parts. Besides, even though the electrospun composites containing $16 \%$ coconut oil and $8 \%$ silver nanoparticles demonstrated the biggest ZOIs. The ZOIs found with this fiber were $20.0 \pm 0.2 \mathrm{~mm}$ against E. coli and $14.8 \pm 0.4 \mathrm{~mm}$ against $S$. aureus.

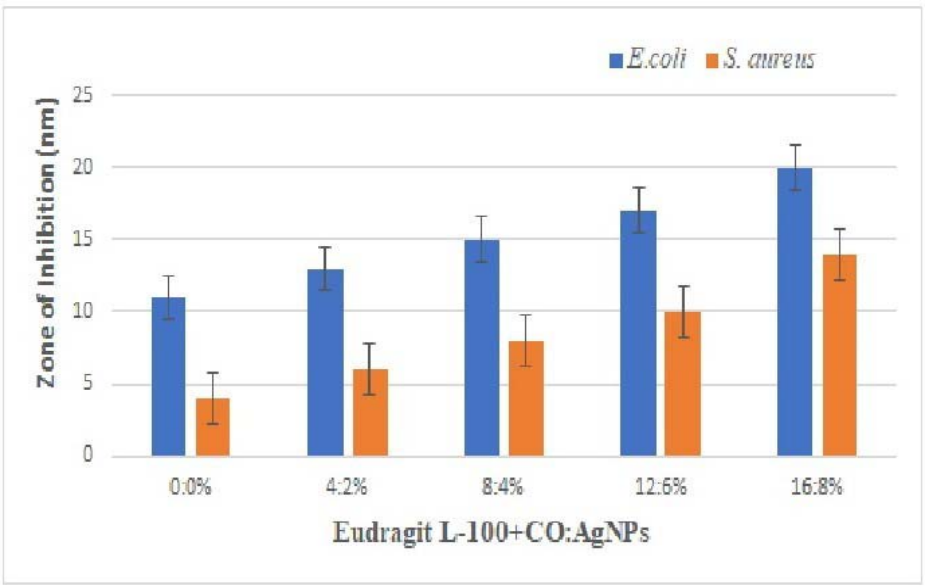

Fig. 9: Zones of inhibition (ZOI) of nanofiber containing diff. concentrations of CO and AgNPs with Eudragit L-100 against $E$. coli and $S$. aureus using the disk diffusion method, ${ }^{*}$ AgNPs= Silver Nanoparticles; $C O=C o c o n u t ~ o i l, ~ m e a n \pm S D ~(n=3)$ 


\section{CONCLUSION}

It is concluded that the examination shows the nanofibrous dressing created for Eudragit L-100 that contains coconut oil and silver nanoparticles having disinfectant properties between E. coli and $S$. aureus in vitro contemplates. Coconut oil diminished inflexibility for electrospun filaments through dissemination and penetration, altering the lipophilicity. CEF refined the complex nanofiber demonstrated noteworthy development and multiplication uncovered with the MTT test. Cells obsession ponders demonstrated the fibroblast developed the characteristic structure of fiber mat which contains coconut oil and silver nanoparticles, in contrast, with circular morphological behavior embraced with electrospun nanofiber, in view with solid lipophilicity. Hindering the obstructions in presence of injury dressing, the fiber dressing is created for cutting edge morphology by typifying coconut oil into strands utilizing an electrospinning strategy.

\section{FUNDING}

Nil

\section{AUTHORS CONTRIBUTIONS}

All authors have contributed equally.

\section{CONFLICT OF INTERESTS}

The author has no conflict of interest to declare.

\section{REFERENCES}

1. P Zahedi, I Rezaeian, SO Ranaei Siadat, SH Jafari, P Supaphol. A review on wound dressings with an emphasis on electrospun nanofibrous polymeric bandages. Polym Adv Technol 2010;21:77-95.

2. Z Yang, H Peng, W Wang, T Liu. Crystallization behavior of poly( $\varepsilon$-caprolactone)/layered double hydroxide nanocomposites. J Appl Polym Sci 2010;116:2658-67.

3. HS Sofi, R Ashraf, AH Khan, MA Beigh, S Majeed, FA Sheikh. Reconstructing nanofibers from natural polymers using surface functionalization approaches for applications in tissue engineering, drug delivery and biosensing devices. Mater Sci Eng C 2019;94:1102-24.

4. X Zhang, Y Lu. Centrifugal spinning: an alternative approach to fabricate nanofibers at high speed and low cost. Polym Rev 2014;54:677-701.

5. S Mahalingam, M Edirisinghe. Forming of polymer nanofibers by a pressurised gyration process. Macromol Rapid Commun 2013;34:1134-9.

6. J Mahavir, L Sneh, K Preeti, M Tulika. Application of nanostructures in antimicrobial therapy. Int J Appl Pharm 2018;10:11-25.

7. S Patel, A Maheshwari, A Chandra. Microwave-assisted synthesis of AGnP using aqueous leaves extract of Vinca rosea and its therapeutic application. Int J Pharm Pharm Sci 2015;7:254-8.
8. A Chandra, S Garg. Effect of varying concentration of herbal extract of nyctanthes arbor-tristis leaf on synthesis of silver nanoparticles and its evaluation. Int J Pharm Pharm Sci 2015;7:143-7.

9. SJ Lee. Electrospun chitosan nanofibers with controlled levels of silver nanoparticles preparation, characterization and antibacterial activity. Carbohydr Polym 2014;111:530-7.

10. A Prashar, IC Locke, CS Evans. Cytotoxicity of lavender oil and its major components to human skin cells. Cell Prolif 2004;37:221-9.

11. IF Efremov. The dilatancy of colloidal structures and polymer solutions. Russ Chem Rev 1982;5:160-77.

12. K Balasubramanian, KM Kodam. Encapsulation of therapeutic lavender oil in an electrolyte assisted polyacrylonitrile nanofibres for antibacterial applications. RSC Adv 2014;4:54892-901.

13. DJ Daferera, PA Tarantilis, MG Polissiou. Characterization of essential oils from lamiaceae species by fourier transform Raman spectroscopy. J Agric Food Chem 2002;50:5503-7.

14. I Samfira, S Rodino, P Petrache, RT Cristina, M Butu, M Butnariu. Characterization and identity confirmation of essential oils by mid infrared absorption spectrophotometry. Dig J Nanomater Biostructures 2015;10:557-66.

15. T Velnar, T Bailey, V Smrkolj. The wound healing process: an overview of the cellular and molecular mechanisms. J Int Med Res 2009;37:1528-42.

16. K Webb, V Hlady, PA Tresco. Relative importance of surface wettability and charged functional groups on NIH 3T3 fibroblast attachment, spreading, and cytoskeletal organization. J Biomed Mater Res 1998;41:422-30.

17. MS Khan, GD Vishakante. Development and evaluation of porous chitosan nanoparticles for treatment of enterotoxigenic Escherichia coil infection. J Biomed Nanotechnol 2013;9:107-14.

18. KP Chellamani, RSV Balaji, D Veerasubramanian, J Sudharsan. Wound healing ability of herbal drug incorporated PCL (Poly $(\varepsilon-$ caprolactone)) wound dressing. J Acad Ind Res 2014;2:622-6.

19. M Rafiq, T Hussain, S Abid, A Nazir, R Masood. Development of sodium alginate/PVA antibacterial nanofibers by the incorporation of essential oils. IOP Sci 2018;5:5-9.

20. A Ghavami Nejad. Mussel-inspired electrospun nanofibers functionalized with size-controlled silver nanoparticles for wound dressing application. ACS Appl Mater Interfaces 2015; 7:12176-83.

21. FA Sheikh. Electrospun antimicrobial polyurethane nanofibers containing silver nanoparticles for biotechnological applications. Macromol Res 2009;17:688-96.

22. M Abrigo, SL McArthur, P Kingshott. Electrospun nanofibers as dressings for chronic wound care: advances, challenges, and future prospects. Macromol Biosci 2014;14:772-92.

23. CA Dos Santos. Silver nanoparticles: therapeutical uses, toxicity, and safety issues. J Pharm Sci 2014;103:1931-44.

24. P Fomby. Stem cells and cell therapies in lung biology and diseases: conference report. Ann Am Thorac Soc 2010;12:181204. 